reward-relevant substrates. Most notably, mesocorticolimbic dopamine circuitry is uniquely sensitive to disruption by drugs in adolescence, resulting in lasting behavioral changes linked to addiction vulnerability [1].

Our recent work in rodents highlights a novel role for guidance cues in (a) the adolescent establishment of mesocorticolimbic dopamine connectivity and cognitive processing and (b) the enduring effects of drugs of abuse on these events. First, we discovered that dopamine axons still grow to the prefrontal cortex (PFC) in adolescence [2]. This is the first concrete demonstration of long-distance axon growth in late postnatal development, which entails targeting decisions by dopamine axons en route to their final postsynaptic partners. Axon targeting relies on the interaction of extracellular guidance cues and their receptors. We find that the guidance cue Netrin-1 and its receptor DCC dictate dopamine axon targeting in adolescence. Specifically, mesolimbic dopamine axons have high levels of DCC and recognize the nucleus accumbens as their final target in adolescence. In contrast, mesocortical dopamine axons have little or no DCC and therefore fail to recognize this region as their final target and grow to the PFC instead. Reduced DCC expression in mesolimbic dopamine axons induces targeting errors in the nucleus accumbens and their ectopic growth to the PFC. By segregating dopamine innervation to cortical or noncortical regions, DCC receptors organize PFC structure and function, including cognitive behaviors that are altered in addiction [2].

Second, we demonstrated that repeated non-contingent exposure to amphetamine in adolescence, at a dose resembling human recreational use, downregulates DCC expression in dopamine neurons [3]. This perfectly positions DCC signaling to mediate the enduring neuroanatomical and behavioral consequences of adolescent drug use. Indeed, amphetamine in early adolescence leads to an increase in the span of dopamine innervation to the PFC. However, it also leads to disorganized synaptic contacts and reduced dopamine turnover in adulthood $[4,5]$. These alterations, in turn, produce deficits in behavioral inhibition and exaggerated salience attribution to drug-paired contexts; two behaviors associated with addiction susceptibility $[4,5]$.

Our findings indicate that DCC signaling wires the adolescent PFC and contributes to amphetamine-induced susceptibility to addiction. Interestingly, amphetamine downregulates DCC via micro-RNAs, which are important biomarkers and mediators of psychiatric conditions [3]. Furthermore, variations in DCC expression occur in humans and lead to altered mesocorticolimbic connectivity [6]. Our work therefore contributes a novel perspective to the ongoing efforts of developing prevention and treatment strategies for addiction. The DCC pathway represents a promising site for targeted intervention during adolescence both to counteract detrimental effects of early drug use and promote healthy brain development.

\section{FUNDING}

This work was supported by the National Institute on Drug Abuse (R01DA037911 to C.F.; F31DA041188 to L.M.R.), the Canadian Institutes of Health Research (MOP-74709 to C.F.), and the Natural Sciences and Engineering Research Council of Canada (2982226 to C.F.). C.F. is a research scholar of the Fonds de Recherche du Québec Santé. L.M.R. was supported by predoctoral fellowships from The Djavad Mowafaghian Foundation and Fulbright Canada.Competing interest: The authors declare that they have no competing interests.

Publisher's note: Springer Nature remains neutral with regard to jurisdictional claims in published maps and institutional affiliations.

\section{REFERENCES}

1. Gulley JM, Juraska JM (2013). The effects of abused drugs on adolescent development of corticolimbic circuitry and behavior. Neuroscience https://doi.org/ 10.1016/j.neuroscience.2013.05.026.

2. Reynolds LM, Pokinko M, Torres-Berrío A, Cuesta S, Lambert LC, Del Cid-Pellitero $E$, et al. DCC receptors drive prefrontal cortex maturation by determining dopamine axon targeting in adolescence. Biol Psychiatry. 2018a;83:181-92.

3. Cuesta S, Restrepo-Lozano JM, Silvestrin S, Nouel D, Torres-Berrío A, Reynolds LM, et al. Non-contingent exposure to amphetamine in adolescence recruits miR-218 to regulate Dcc expression in the VTA. Neuropsychopharmacology. 2018;43:900-11.

4. Reynolds LM, Makowski CS, Yogendran SV, Kiessling S, Cermakian N, Flores C. Amphetamine in adolescence disrupts the development of medial prefrontal cortex dopamine connectivity in a dcc-dependent manner. Neuropsychopharmacology. 2015;40:1101-12.

5. Reynolds LM, Yetnikoff L, Pokinko M, Wodzinski M, Epelbaum JG, Lambert LC, et al. (2018b) Early adolescence is a critical period for the maturation of inhibitory behavior. In Press, Cerebral Cortex

6. Vosberg DE, Zhang $\mathrm{Y}$, Menegaux A, Chalupa A, Manitt C, Zehntner S, et al. Mesocorticolimbic connectivity and volumetric alterations in DCC mutation carriers. J Neurosci. 2018;38:4655-4665.

\title{
The ventromedial prefrontal cortex: a putative locus for trait inattention
}

\author{
Matthew D. Albaugh ${ }^{1}$ and Alexandra S. Potter (iD) \\ Neuropsychopharmacology (2019) 44:226-227; https://doi.org/10.1038/s41386-018-0193-7
}

Efforts to identify structural brain correlates of attention-deficit/ hyperactivity disorder (ADHD) have yielded inconsistent results, with no common structural biomarker emerging across studies. Methodological factors may obscure underlying brain-behavior 
relations in the study of ADHD symptomatology, including the use of categorical diagnoses [1], relying on behavioral ratings from a single informant, and small sample sizes. Indeed, empirically based assessment of psychopathology has revealed aspects of dimensionality with regard to many psychiatric conditions, including ADHD [2]. Further, recent reports suggest that information from multiple informants increases the validity of ADHD assessment [3]. Population-based neuroimaging studies provide the opportunity to more rigorously characterize the neurobiological underpinnings of ADHD symptomatology by using dimensional, multi-informant behavioral ratings, and by examining convergence across multiple assessment modalities-including genetic and neurocognitive domains.

Studying a population-based sample of adolescents $(N=1538$; 785 females) $(M=14.53$ years old, $S D=0.41)$, we investigated the relationship between dimensional measures of ADHD symptomatology, brain structure, and reaction time variability (RTV) - an objective index of attentional lapses [4]. Parent ratings of ADHD symptoms, adolescent self-reports of ADHD symptoms, and RTV were each negatively associated with gray matter volume (GMV) in an overlapping region of the ventromedial prefrontal cortex (vmPFC). To our knowledge, this study represents the largest voxel-based morphometry (VBM) study to date on adolescent ADHD symptomatology. Despite modest correlations between multi-informant behavioral ratings $(r=0.36-0.66)$, we observed striking convergence in the vmPFC with regard to the anatomical correlates of each behavioral measure. Similarly, while RTV was weakly correlated with behavioral ratings of ADHD symptomatology $(r=0.11-0.14)$, there was considerable overlap with regard to anatomical correlates. Given convergence across dimensional, multi-informant behavioral ratings, and a measure of neurocognitive functioning that has been previously tied to ADHD, our findings indicate that vmPFC structure is a brain-based marker for inattention in adolescents.

Utilizing gene expression data collected as part of the Allen Human Brain Atlas [5], we found that the statistical map representing the relationship between GMV and ADHD symptomatology was differentially correlated with patterns of DRD1 and $D R D 2$ gene expression. Our results appear consistent with models of cognitive dysfunction that postulate relative imbalance between D1 and D2 systems; however, it is important to emphasize that these gene expression analyses were meant to be hypothesis-generating in nature. More comprehensive studies of gene expression represent a plausible direction for future research.

The National Institute of Mental Health has placed growing emphasis on the need to, "identify and validate biomarkers and novel treatment targets relevant to the prevention, treatment, and recovery of psychiatric disorders," as evidenced by the new Computational Psychiatry Program (https://www.nimh.nih.gov/ about/organization/dtr/adult-psychopathology-and-psychosocialinterventions-research-branch/computational-psychiatry-program. shtml). An exciting avenue of our research has been to examine the extent to which vmPFC structure during adolescence predicts subsequent symptom trajectories into adulthood. Using data from the 5-year follow-up wave of the IMAGEN study, we found that adolescent vmPFC volume predicts adult ADHD symptomatology while controlling for baseline symptomatology [6]. Intriguingly, these latter results suggest that early structural development of the vmPFC may be consequential for the subsequent expression of hyperactive/inattentive symptoms in adulthood.

\section{ACKNOWLEDGEMENTS}

This work received support from the following sources: the European Union-funded FP6 Integrated Project IMAGEN (Reinforcement-related behaviour in normal brain function and psychopathology) (LSHM-CT- 2007-037286), the FP7 projects IMAGEMEND (602450; IMAging GEnetics for MENtal Disorders), AGGRESSOTYPE (602805) and MATRICS (603016), the Innovative Medicine Initiative Project EU-AIMS (115300-2), the Medical Research Council Grants "Developmental pathways into adolescent substance abuse" (93558) and Consortium on Vulnerability to Externalizing Disorders and Addictions [C-VEDA] (MR/N000390/1), the Swedish funding agencies VR, FORTE, and FORMAS, the Medical Research Council and the Wellcome Trust (Behavioural and Clinical Neuroscience Institute, University of Cambridge), the National Institute for Health Research (NIHR) Biomedical Research Centre at South London and Maudsley NHS Foundation Trust and King's College London, the Bundesministeriumfür Bildung und Forschung (BMBF grants 01GS08152; 01EV0711; eMED SysAlc01ZX1311A; Forschungsnetz AERIAL), the Deutsche Forschungsgemeinschaft (DFG grants $S M$ 80/7-1, SM 80/7-2, SFB 940/1), the National Institutes of Health, USA (Axon, Testosterone and Mental Health during Adolescence; RO1 MH085772-01A1), and by NIH Consortium grant U54 EB020403, supported by a cross-NIH alliance that funds Big Data to Knowledge Centres of Excellence. ASP is supported by P20GM103644 (PI: Stephen T. Higgins), Agency: NIGMS Vermont Center on Behavior and Health. Competing interests: The authors declare no competing interests.

Publisher's note: Springer Nature remains neutral with regard to jurisdictional claims in published maps and institutional affiliations.

\section{REFERENCES}

1. Ducharme S, Hudziak JJ, Botteron KN, Albaugh MD, Nguyen TV, Karama S, et al. Decreased regional cortical thickness and thinning rate are associated with inattention symptoms in healthy children. J Am Acad Child Adolesc Psychiatry. 2012;51:18-27 e.12. https://doi.org/10.1016/j.jaac.2011.09.022.

2. Hudziak JJ, Achenbach TM, Althoff RR, Pine DS. A dimensional approach to developmental psychopathology. Int J Methods Psychiatr Res. 2007;16(Suppl 1): S16-23. https://doi.org/10.1002/mpr.217.

3. Martel MM, Schimmack U, Nikolas M, Nigg JT. Integration of symptom ratings from multiple informants in ADHD diagnosis: a psychometric model with clinical utility. Psychol Assess. 2015;27:1060-71. https://doi.org/10.1037/pas0000088.

4. Albaugh MD, Orr C, Chaarani B, Althoff RR, Allgaier N, D'Alberto N, et al. Inattention and reaction time variability are linked to ventromedial prefrontal volume in adolescents. Biol Psychiatr. 2017 https://doi.org/10.1016/j. biopsych.2017.01.003.

5. Hawrylycz MJ, Lein ES, Guillozet-Bongaarts AL, Shen EH, Ng L, Miller JA, et al. An anatomically comprehensive atlas of the adult human brain transcriptome. Nature. 2012;489:391-9. https://doi.org/10.1038/nature11405.

6. Albaugh MD, Ivanova M, Chaarani B, Orr C, Allgaier N, Althoff RR, et al. Ventromedial prefrontal volume in adolescence predicts hyperactive/inattentive symptoms in adulthood. Cereb Cortex. 2018. https://doi.org/10.1093/cercor/ bhy066. [Epub ahead of print]. 